\title{
Evaluation of the single-frequency operation of a short vertical external-cavity semiconductor laser at $852 \mathrm{~nm}$
}

\author{
Fabíola A. Camargo $^{\mathrm{a}}$, Sylvie Janicot ${ }^{\mathrm{a}}$, Isabelle Sagnes ${ }^{\mathrm{b}}$, Arnaud Garnache ${ }^{\mathrm{c}}$, Patrick Georges ${ }^{\mathrm{a}}$ and \\ Gaëlle Lucas-Leclin ${ }^{\mathrm{a}}$ \\ a Laboratoire Charles Fabry, Institut d'Optique, CNRS, Univ Paris-Sud, \\ 2 avenue Augustin Fresnel, 91127 Palaiseau Cedex, France \\ ${ }^{\mathrm{b}}$ Laboratoire de Photonique et de Nanostructures, CNRS UPR20, \\ Route de Nozay, 91460 Marcoussis, France \\ c Institut d'Electronique du Sud, CNRS UMR5507, Université Montpellier 2, \\ 34095 Montpellier Cedex 5, France
}

\begin{abstract}
We present in this work the study of a short vertical external cavity semiconductor laser in single longitudinal operation at $852 \mathrm{~nm}$ without intracavity elements. Two different configurations were studied, a plane-plane configuration, stabilized by the thermal lens and a plane-concave configuration. The influence of the output coupler transmission and the thermal lens has been studied. In the plane concave configuration we have demonstrated more than $100 \mathrm{~mW}$ in stable single frequency operation using a very compact cavity emitting around $852 \mathrm{~nm}$.
\end{abstract}

Keywords: Semiconductor lasers, VECSEL, Thermal lens, Single-frequency

\section{INTRODUCTION}

Stable single-frequency laser with narrow linewidth emission $(<500 \mathrm{kHz})$, fine tunability over a few $\mathrm{GHz}$ and output power in the $0.1 \mathrm{~W}$ range at $852 \mathrm{~nm}$ is required for the laser sources used in cesium atomic clocks. Until now different approaches have been studied in order to fulfill these properties : single frequency high power lasers have been demonstrated with distributed feedback diode lasers [1] and tapered extended cavity diode lasers [2]. However, these two solutions suffer from beam quality degradation at high power. A possible alternative solution to obtain a compact and simple single frequency source with narrow linewidth and good beam quality is an optically-pumped semiconductor vertical external-cavity surface-emitting laser (OPS-VECSEL) [3]. OPS-VECSEL's combine the benefits of solid-state lasers and laser diodes. It has the advantage of using a semiconductor as the gain medium which allows versatility in the wavelength emission. Moreover single-frequency, narrow linewidth, emission is obtained in simple and compact setups.

Watt-level, single frequency emission was demonstrated from OPS-VECSEL's at $1 \mu \mathrm{m}[4,5]$, however few works have been done at $852 \mathrm{~nm}[6,7]$. Actually at this wavelength, GaAs/AlGaAs quantum wells (QW's) are used as the active layers, which present a lower optical gain; furthermore the high content of Aluminium in the barriers and in the Bragg mirror makes the semiconductor structure more sensitive to degradation. To date, output power in the $0.5 \mathrm{~W}$ range has been demonstrated under multilongitudinal mode operation, and the output power drops drastically in single-frequency operation due to the introduction of intracavity elements. Following the approach described in Ref. 5, we investigate in this work the single-frequency emission of OPS-VECSEL's at $852 \mathrm{~nm}$ in short external-cavities without intracavity elements.

\section{DESIGN OF A SHORT-CAVITY SINGLE-FREQUENCY VECSEL}

Our aim is to develop a single-frequency VECSEL in the simplest possible configuration, without intracavity wavelength-selective filter and in a plane-plane cavity. This configuration was proposed in Ref. 8, and a stable singlefrequency, narrow linewidth emission with output power as high as $2.1 \mathrm{~W}$ at $1 \mu \mathrm{m}$ was demonstrated [5]. The proper 
design of such a laser source implies in one hand to get a clear understanding of the dynamics of the single-frequency operation, and on the other hand to evaluate the pump-induced thermal lens inside the structure, which contributes to the stabilization of the laser cavity.

\subsection{Description of the active structure}

The semiconductor structure which has been studied in this work is described in Figure 1; it has been grown by metalorganic chemical-vapor deposition on a $350 \mu \mathrm{m}$-thick GaAs substrate. It is designed for emission at $\lambda_{\mathrm{L}}=852 \mathrm{~nm}$ under barrier-pumping at $\lambda \leq 700 \mathrm{~nm}$. The $30 \lambda / 4$-thick active region contains seven $8 \mathrm{~nm}$-thick GaAs QW's embedded within $\mathrm{Al}_{22 \%} \mathrm{Ga}_{78 \%}$ As barriers, which absorb $\eta_{\mathrm{abs}}=86 \%$ of the incident pump power. The QW's are distributed among the optical standing-wave antinodes positions with a repartition 1-1-1-1-0-1-0-1-0-0-1 (from the top surface) which is calculated in order to provide a nearly constant excited carrier density among them. Two $30 \mathrm{~nm}$-thick $\mathrm{Al}_{39 \%} \mathrm{Ga}_{61 \%} \mathrm{As}$ produce a potential barrier on each side of the active region for carrier confinement. The high-reflectivity Bragg mirror consists in 32.5 pairs of $\mathrm{Al}_{22} \mathrm{Ga}_{78 \%} \mathrm{As} / \mathrm{AlAs}$ layers, and absorbs the remaining pump power. A $20 \mathrm{~nm}$-thick $\mathrm{In}_{48 \%} \mathrm{Ga} 52 \% \mathrm{P}$ protects the top surface of the structure from oxidation of the Al-rich barriers. Though in the following experiments the laser output power will be limited by the strong thermal roll-over induced by the low conductivity of the GaAs substrate, it should still be compatible with the requirements for metrology applications.

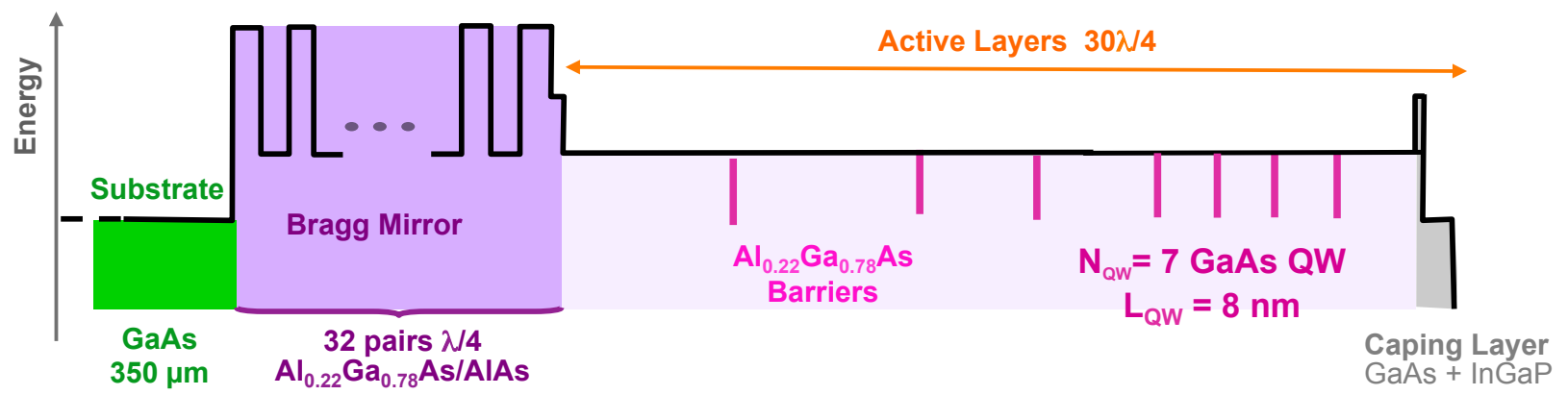

Figure 1 : Description of the active structure.

\subsection{Single-frequency dynamical behavior}

The active structure of a OPS-VECSEL has a nearly-ideal homogenous gain, which implies that the spectral bandwidth of the laser emission is simply driven by mode competition [9]. The narrowing of the laser bandwidth $\Delta v_{\mathrm{L}}$ with time, assuming a parabolic gain spectral profile, is described by $\Delta v_{L}(t)=\Delta v_{\text {gain }} \sqrt{\ln 2 \times \tau_{p h} / t}$, with $\Delta v_{\text {gain }}$ the gain bandwidth, $\tau_{p h}=2 L_{\text {ext }} /(c \times L)$ the intracavity photon lifetime, $L_{\text {ext }}$ the cavity length and $L$ the total optical losses (output coupler and intracavity losses). When $\Delta v_{L}$ decreases below the free spectral range of the laser cavity, the laser collapses into one single longitudinal mode; the corresponding characteristic time $t_{c}$ for the single-frequency operation of the OPSVECSEL is thus:

$$
t_{c}=\frac{4 \ln 2}{c \lambda_{L}^{4}} \times \frac{L_{e x t}^{3} \Delta v_{\text {gain }}^{2}}{L}
$$

Experimentally, a stable single-frequency operation of a OPS-VECSEL will be obtained if $t_{c}$ is shorter than any typical technical fluctuations time (thermal and mechanical noise, or pump intensity fluctuations) of the laser cavity; then, after a perturbation arises, the laser shortly goes back to a single-frequency emission. Two approaches are possible to significantly decrease $t_{c}$, either to decrease the gain bandwidth $\Delta v_{\text {gain }}$ by introducing wavelength-selective filters (etalon, birefringent filter), or to reduce the cavity length $L_{e x t}$. The first one is most commonly used, as it is simpler to implement, provides a wide wavelength tunability and allows the introduction of additional components (non-linear crystals, ...). Nevertheless stable single-frequency operation of a short cavity OPS-VECSEL without intracavity wavelength filter has already been demonstrated [5,7]. Here we investigate this solution for the realization of a single-frequency OPSVECSEL emitting at $852 \mathrm{~nm}$; with a cavity length $L_{\text {ext }}=5 \mathrm{~mm}$, optical losses $L$ of about $3 \%$, a gain bandwidth $\Delta v_{\text {gain }}=$ 
$20 \mathrm{~nm}$, the characteristic time $t_{c}$ should be about $60 \mu \mathrm{s}$, which is actually below any typical fluctuations time (in the ms range), and is equivalent to the value calculated with a $L_{e x t}=25 \mathrm{~mm}$ long cavity and an intracavity filter with a bandwith $\Delta v_{\text {gain }}=2 \mathrm{~nm}$.

\subsection{Evaluation of the thermal lens}

Optical pumping of a gain medium, either semiconductor or crystal, induces a local increase of its temperature because of the amount of absorbed pump power that is converted into heat. The thermal fraction $\eta_{t h}$ is the amount of incident pump power actually converted into heat, and is intrinsically limited by the quantum defect between the pump and the laser photon energies and the non-radiative transitions. Additionally in the active structure of a OPS-VECSEL, residual absorption of the pump power outside the gain region (Bragg mirror, substrate), also contributes to the heating of the active region. The thermal fraction is thus expressed as $\eta_{t h}=1-\eta_{a b s} \eta_{r a d} \lambda_{P} / \lambda_{L}$, with $\eta_{a b s}$ the pump absorption in the gain medium, $\lambda_{P}$ and $\lambda_{L}$ the pump and laser wavelengths respectively, and $\eta_{\text {rad }}$ the radiative efficiency. In our structure, $\eta_{\text {th }}$ is evaluated to $\sim 50 \%$ without laser emission, and $\sim 35 \%$ with laser emission.

In order to evaluate the temperature inside the semiconductor structure, we have first considered an analytical approach. Assuming a top-hat pump profile (waist radius $W_{p}$ ) and a bottom-side cooling, the maximum temperature rise, at the top surface of the structure, is estimated as $\Delta T_{\max } \cong \eta_{t h} R_{t h} P_{p}$, with $P_{p}$ the incident pump power and $R_{t h}$ the thermal resistance of the semiconductor structure. The latter is evaluated assuming a 1D-longitudinal cooling within the thin layers of the active region and Bragg mirrors (thickness $e_{i}$, thermal conductivity $\kappa_{i}$ ), and a 3D cooling in the GaAs substrate; this hypothesis is valid as long as the pump waist radius $W_{p}$ is much smaller than the substrate thickness:

$$
R_{t h}=\frac{1}{\pi W_{P}^{2}} \sum_{i} \frac{e_{i}}{\kappa_{i}}+\frac{1}{\kappa_{s} \pi W_{P}}
$$

From this expression, we estimate a thermal resistance $R_{t h}=230 \mathrm{~K} / \mathrm{W}$ for a $W_{p}=35 \mu \mathrm{m}$ pump spot, with as expected a major contribution (70\%) from the GaAs substrate. For $\eta_{t h} \times P_{p}=0.3 \mathrm{~W}$ of thermal power dissipated into the structure, the maximum temperature rise is thus $\Delta T_{\max }=71 \mathrm{~K}$. This analytical estimation has been compared to the value resulting from the numerical resolution of the heat transfer equation in the structure using a finite-element method (COMSOL Multiphysics). For this simulation, the semiconductor structure was described by three layers - active region, Bragg mirror, GaAs substrate - glued onto a copper heatsink with a $50 \mu \mathrm{m}$-thich heatpaste. The parameters of the simulation (layer thickness and conductivity) are given in Table 1 . The cooper heatsink temperature is set to $15^{\circ} \mathrm{C}$, and a cylindrical symmetry of the whole system is assumed. The temperature distribution inside the structure is shown in Figure 2. The temperature increase is maximum in the active region and reaches $\Delta T_{\max }=59 \mathrm{~K}$, which is in relative good agreement with our analytical estimation. The temperature distribution extends over a diameter of $\sim 500 \mu \mathrm{m}$. The on-axis temperature remains almost constant among the active region $(\Delta T(r=0)=7 \mathrm{~K})$. The main temperature difference is created inside the substrate, as expected from the analytical evaluation of the thermal resistance.

\begin{tabular}{|r|c|c|}
\hline & Thickness & Equivalent thermal conductivity \\
\hline Active region & $1.7 \mu \mathrm{m}$ & $20 \mathrm{Wm}^{-1} \mathrm{~K}^{-1}$ \\
\hline Bragg mirror & $4.3 \mu \mathrm{m}$ & $34 \mathrm{Wm}^{-1} \mathrm{~K}^{-1}$ \\
\hline GaAs substrate & $350 \mu \mathrm{m}$ & $55 \mathrm{Wm}^{-1} \mathrm{~K}^{-}$ \\
\hline Thermal heatpaste & $50 \mu \mathrm{m}$ & $2 \mathrm{Wm}^{-1} \mathrm{~K}^{-1}$ \\
\hline Copper heatspreader & $5 \mathrm{~mm}$ & $400 \mathrm{Wm}^{-1} \mathrm{~K}^{-1}$ \\
\hline
\end{tabular}

Table 1: Parameters used for the calculation of the temperature map inside the structure. 
A 346.93

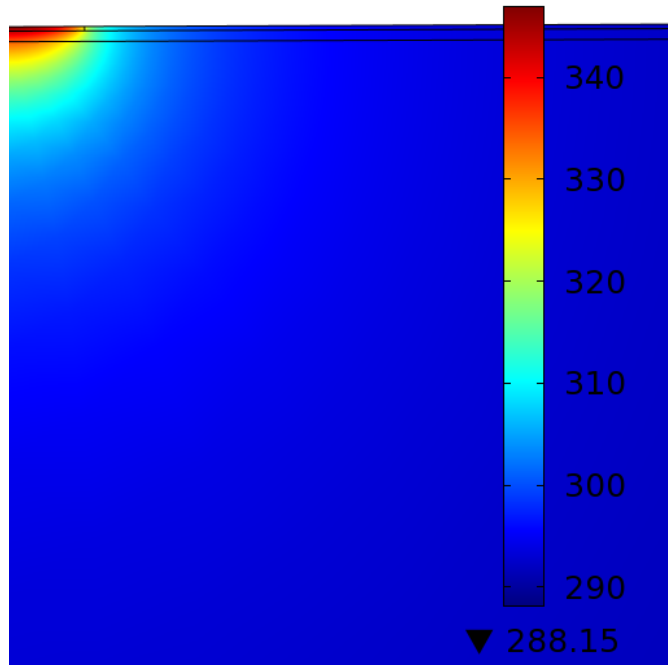

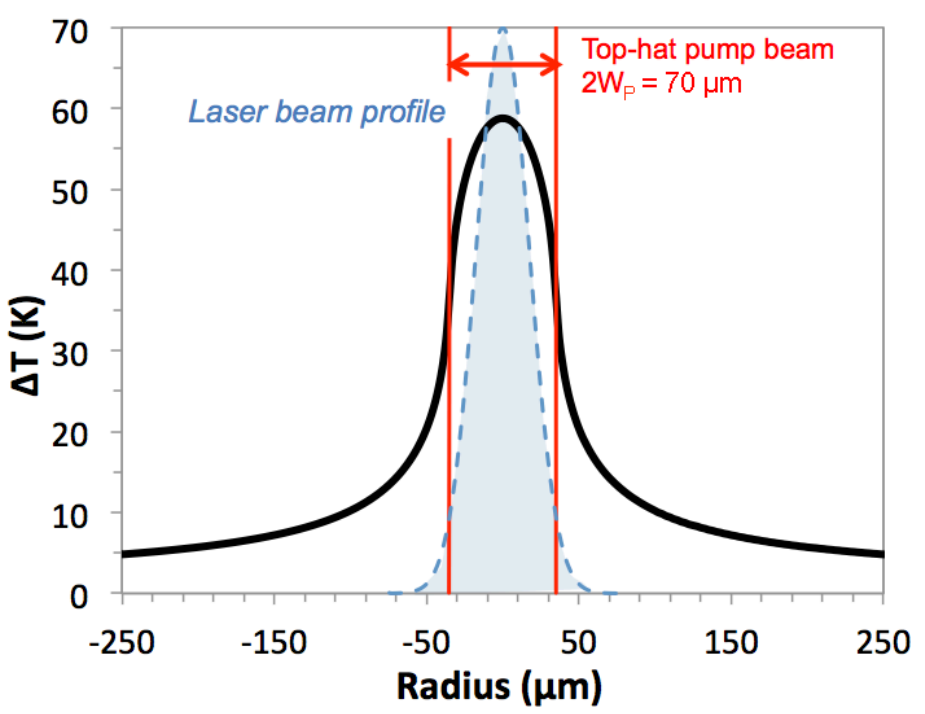

Figure 2: Left - Temperature distributions inside the active structure through the various layers for the structure on GaAs substrate under optical pumping $\left(\eta_{t h} \times P_{p}=0.3 \mathrm{~W}, W_{P}=35 \mu \mathrm{m}\right)$; Right - Radial temperature profile (black line) at the top surface of the structure, and laser beam profile (dashed blue line).

The temperature radial gradient inside the gain medium generates a thermal lensing through different mechanisms: refractive index gradient, either directly thermally-induced or indirectly via the photoelastic effect, and bulging of the end faces resulting from thermo-mechanical stress [10]. The major contribution in semiconductor-based active medium is the thermo-optic refractive index change [11], which depends on the thermal conductivity $\kappa$ of the gain material, on its thermo-optic coefficient $\mathrm{dn} / \mathrm{dT}\left(\sim 2.7 \times 10^{-4} \mathrm{~K}^{-1}\right.$ for the AlGaAs family) and on the cooling system configuration. The optical path difference experienced by the laser beam at $\lambda_{\mathrm{L}}=852 \mathrm{~nm}$ on one roundtrip in the active structure is expressed as:

$$
\begin{aligned}
\Delta(r) & =O P D(0)-O P D(r)=\left(\frac{d n}{d t}\right) \times \int_{0}^{2 d}[T(0, z)-T(r, z)] d z \\
& \cong 2\left(\frac{d n}{d t}\right) d_{\text {lens }} \Delta T(r, z=0)
\end{aligned}
$$

The effective thickness $d_{\text {lens }}$ of the thermal lens is the laser penetration depth within the structure, which is estimated to $d_{\text {lens }} \sim 3.1 \mu \mathrm{m}$ from the calculated laser field intensity profile along the layered structure. As the temperature does not change significantly inside the active structure over this thickness, the temperature distribution on the top layer of the structure will be simply considered for the evaluation of $\Delta(r)$. The radial profile of the optical path difference $\Delta(r)$ is simply deduced from the temperature profile, and a polynomial fit of its variation with radius is done. On the laser waist - approximately equal to the pump beam waist $2 W_{P}$ in our experiments - it exhibits a strong parabolic contribution associated with the thermal lens, but also a significant contribution of the $3^{\text {rd }}$ order spherical aberration (Figure 3 ).

$$
\Delta\left(r \leq W_{P}\right) \cong \frac{r^{2}}{2 f_{t h}}+C \frac{r^{4}}{W_{P}^{4}}
$$

For a thermal power $P_{t h}=0.3 \mathrm{~W}$ dissipated within the active structure (corresponding to an incident pump power of $0.6 \mathrm{~W}$, below the laser threshold) and a $W_{P}=35 \mu \mathrm{m}$ top-hat pump beam profile, the focal length of the thermal lens is evaluated to $f_{\text {th }} \cong 40 \mathrm{~mm}$, which should correspond to the shortest focal length under our experimental conditions (see $\S 3$ ). The thermal lens $f_{t h}$ varies inversely proportionally to the heat power $\eta_{t h} \times P_{p}$ and the thermal resistance of the structure $R_{t h}$. 


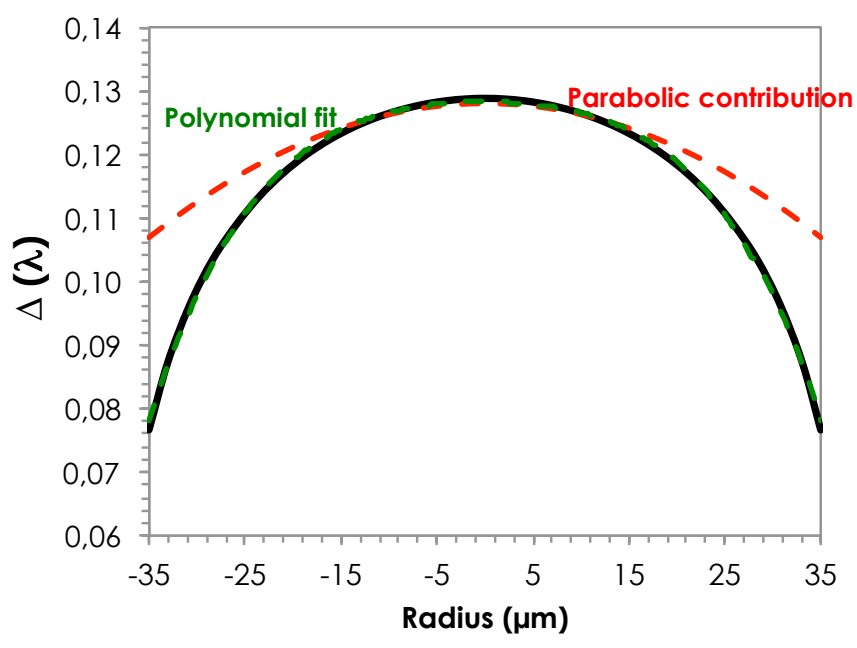

Figure 3: Optical path difference experienced by the laser beam, on one roundtrip inside the laser cavity.

\subsection{Cavity stability}

We have investigated two laser cavities for the short-cavity OPS-VECSEL, with either a plane or a concave $(R=25 \mathrm{~mm})$ output coupler. The cavity stability of these two configurations has been studied using the ABCD matrix formalism, and the laser waist radius $W_{L}$ on the active structure has been calculated as a function of the thermal lens $f_{t h}$ (i.e. the pump power) and of the cavity length $L_{\text {ext }}$. It follows the standard relationship for a 2-mirror cavity [12] :

$$
W_{L}=\sqrt{\frac{L_{e x t} \lambda}{\pi} \sqrt{\frac{g_{2}}{g_{1}\left(1-g_{1} g_{2}\right)}}}
$$

where $g_{1}=1-L_{\text {ext }} / f_{\text {th }}$ and $g_{2}=1-L_{\text {ext }} / R_{2}, f_{\text {th }}$ is the thermal lens focal length and $R_{2}$ is the curvature radius of the output coupler. With no thermal lens and a cavity length $L_{e x t}=1 \mathrm{~mm}$, the plane-concave cavity is stable and the laser waist $W_{L}$ is $36 \mu \mathrm{m}$. For minimum absorption losses, the laser waist radius $W_{L}$ should be smaller than the pump waist radius $W_{P}$. In the following experiments, the pump waist is chosen as $W_{P}=36 \mu \mathrm{m}$. From Figure 4 , the plane-plane cavity is thus expected to operate for strong thermal lenses and short cavities, whereas the concave-plane setup is less sensitive to the thermal lens value for $f_{t h} \geq 40 \mathrm{~mm}$.
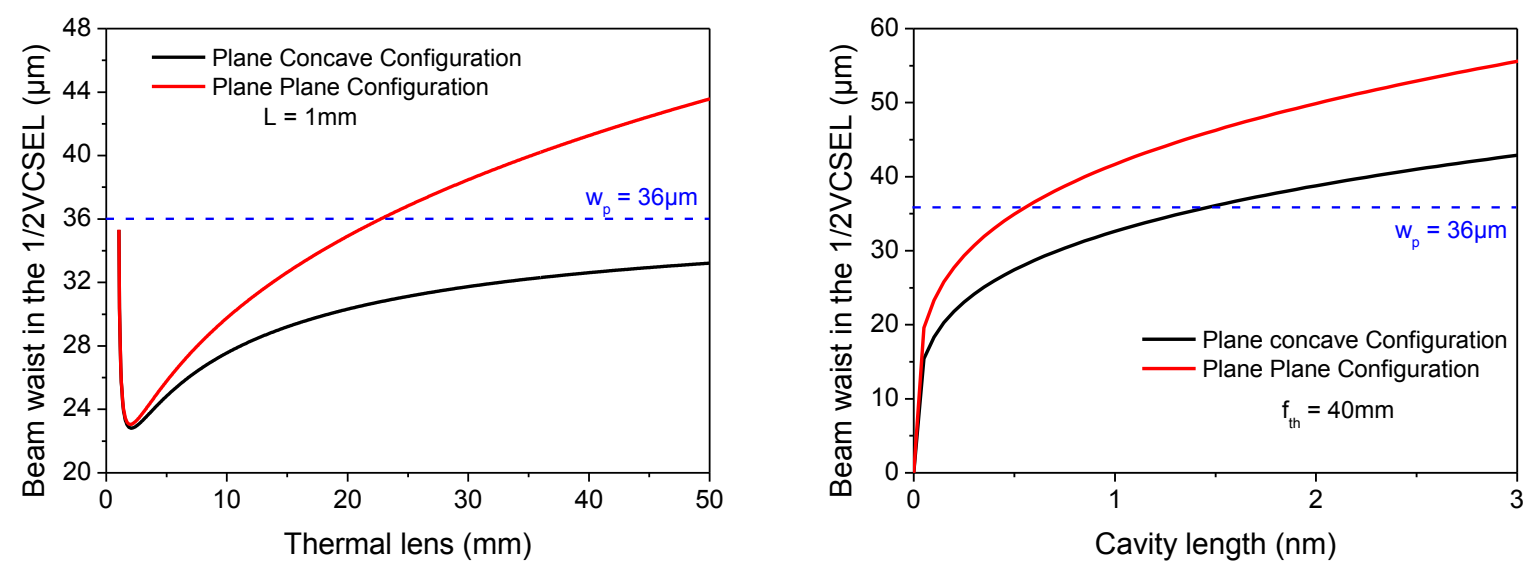

Figure 4: Laser beam waist in the semiconductor structure with the focal length of the thermal lens for a $1 \mathrm{~mm}$ long cavity (left) and with the cavity length for a thermal lens $f_{t h}=40 \mathrm{~mm}$ (right). 


\section{EXPERIMENTAL CHARACTERISATION OF THE LASER EMISSION}

\subsection{Experimental setup}

The active structure used for these experiments is described in section $\$ 2.1$; it is glued with heatpaste on a cooper heatsink cooled by a Peltier element. The chip temperature is maintained at $15^{\circ} \mathrm{C}$ for all the following experiments. The pump source is a $5 \mathrm{~W} 690 \mathrm{~nm}$ fiber-coupled diode laser focused on the semiconductor using two doublets with focal distances of $60 \mathrm{~mm}$ and $40 \mathrm{~mm}$, producing a pump beam waist $W_{P}=36 \mu \mathrm{m}$ in the chip. In order to obtain a good overlap between the pump and the laser beams and to ensure a circular laser beam, the pump beam is nearly perpendicular to the active structure; a slight angle between them prevents parasitic feedback between the pump fiber end and the laser. A dichroic mirror with high transmission at $690 \mathrm{~nm}$ and high reflectance at $850 \mathrm{~nm}$ is placed between the two doublets in order to separate the pump and the laser beams (Figure 5). Different output couplers with transmissions $\mathrm{T}=0.7 \%, 2 \%$ and $3 \%$ are used; the radius of curvature of the concave output coupler is $25 \mathrm{~mm}$.
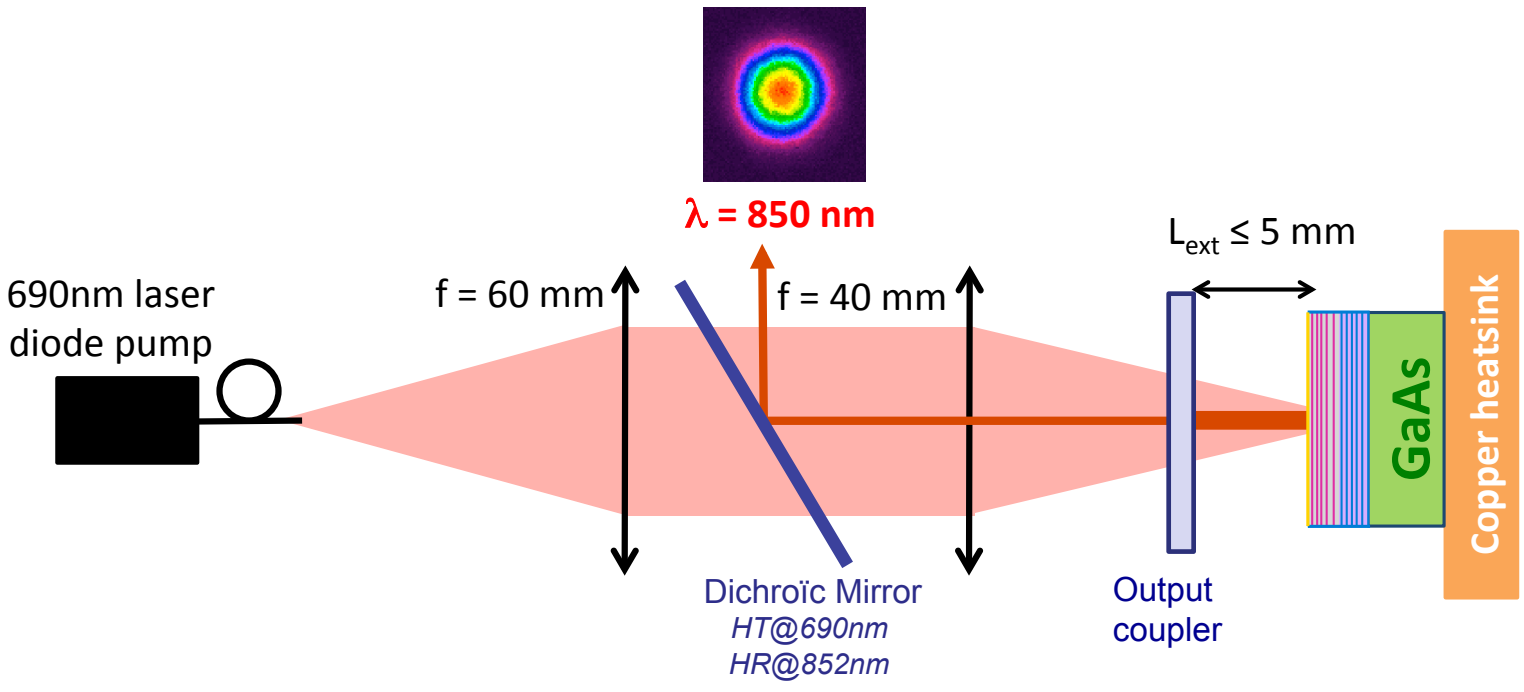

Figure 5: (left) Experimental setup for the short cavity OPS-VECSEL; (insert) Laser beam intensity map, demonstrating a fundamental transverse mode emission.

\subsection{Output power}

In the plane-plane configuration, the maximum output power obtained is $95 \mathrm{~mW}$ for a $3 \%$ transmission (Figure 6). The laser threshold increases with the output coupler transmission $\mathrm{T}$, from an incident pump power of $0.22 \mathrm{~W}\left(\sim 5 \mathrm{~kW} / \mathrm{cm}^{2}\right)$ with $\mathrm{T}=0.7 \%$ to $0.36 \mathrm{~W}\left(\sim 9 \mathrm{~kW} / \mathrm{cm}^{2}\right)$ with $\mathrm{T}=3 \%$. Moreover the slope efficiency of the laser increases and reaches $61 \%$ for $\mathrm{T}=3 \%$. Whatever the output coupler, the thermal roll-over occurs at an incident pump power of about $0.55 \mathrm{~W}$. It is noteworthy that a stable laser operation of the plane-plane cavity is obtained at lower pump powers than expected from our study of the cavity stability based on the thermal lens $(\S 2.4)$ : the focal length of the thermal lens at the incident pump power of $0.22 \mathrm{~W}$ is $f_{t h} \sim 120 \mathrm{~mm}$, corresponding to a laser waist approximately equal to $W_{L}=55 \mu \mathrm{m}$. As already mentioned in similar experiments [5,11] and microchip solid-state lasers, gain-guiding of the laser emission may significantly contribute to the stability of the laser mode.

With the concave output coupler, a maximum output power of $102 \mathrm{~mW}$ is obtained for a transmission $\mathrm{T}=3 \%$. Though the output power is similar in both setups, the laser threshold in this case is $0.15 \mathrm{~W}\left(\sim 4 \mathrm{~kW} / \mathrm{cm}^{2}\right)$; it is three times smaller in the plane-concave configuration, as the stability of the laser cavity is marginally dependent of the pump power contrary to the plane-plane configuration. In both configurations, the laser oscillates in the fundamental transverse mode (Figure 5 , right), and is linearly polarized thanks to a slight gain anisotropy of our semiconductor structure. 

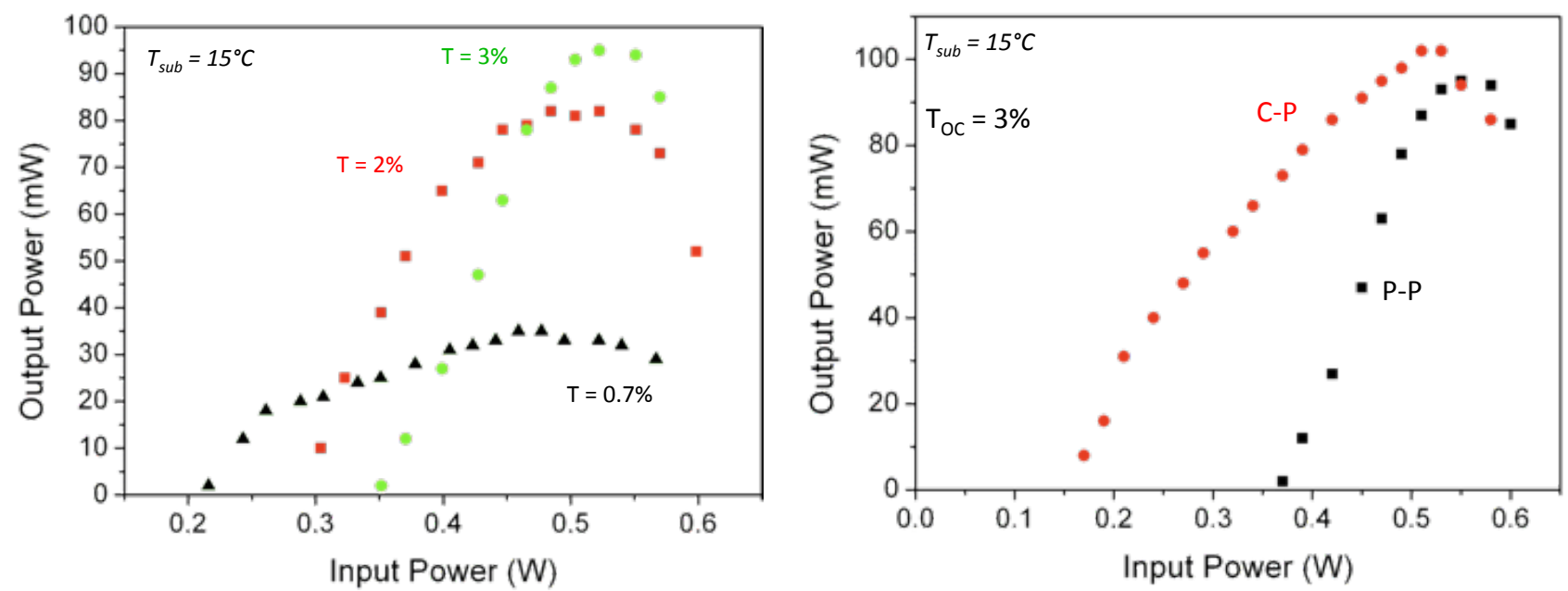

Figure 6: (left) Laser output power as function of the input power in the plane-plane configuration for different output couplers transmissions; (right) Comparison of the laser performance between the two configurations (C-P : concave output coupler; P-P : plane output coupler) used for an output coupler transmission of $3 \%$.

\subsection{Characterization of the laser spectrum}

The spectrum is measured with an optical spectrum analyzer with a resolution of $10 \mathrm{pm}$, much smaller than the free spectral range of the laser, which is about $0.18 \mathrm{~nm}$ for a cavity length $L_{e x t}=2 \mathrm{~mm}$. On a wide range of operating parameters (cavity length $L_{\text {ext }}$, incident pump power), the laser spectrum is single-frequency (Figure 7), with a side-mode suppression ratio $>35 \mathrm{~dB}$. This confirms that single-frequency operation of a VECSEL can be obtained with a short cavity without any spectrally-selective intracavity element, thanks to the reduction of the characteristic time $t_{c}(\S 2.2)$.

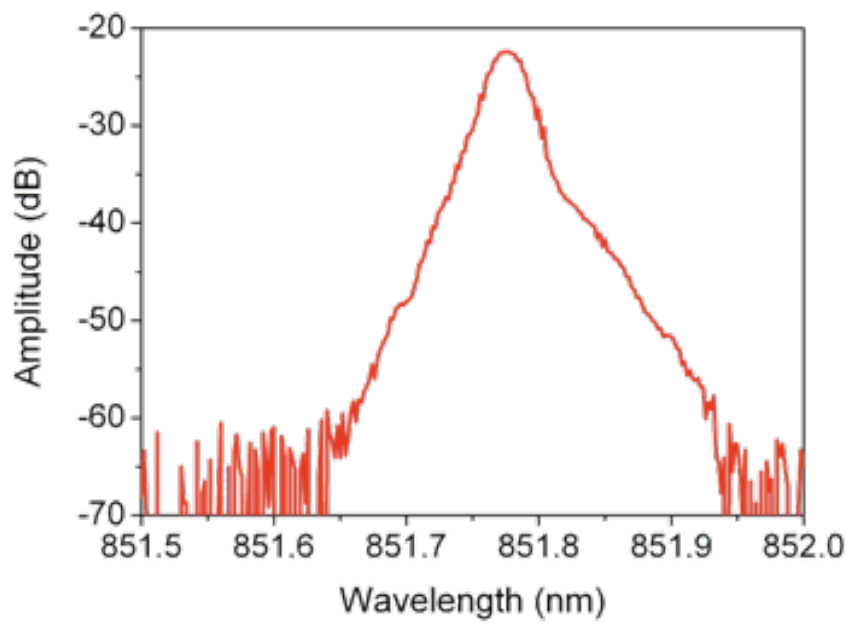

Figure 7: Single frequency laser emission in the plane-plane configuration.

We have finally characterized the stability of the laser line with a wavemeter; the wavelength is recorded every $200 \mathrm{~ms}$ during one hour. The relative variations of the laser wavelength are measured for the two cavity configurations, and with different output couplers. The laser line appears to be more stable for output couplers with a higher transmission, as expected from the expression of $t_{c}$. Actually with the $\mathrm{T}=0.7 \%$ plane output coupler, the laser emission remains singlefrequency on a $20 \mathrm{mn}$ time scale, but its wavelength exhibits a slow thermal shift with large instabilities in the neighborhood of a mode-hopping, every $0.18 \mathrm{~nm}$. With the $\mathrm{T}=3 \%$ plane output coupler, the fluctuations of the laser line remains $\sim 9 \mathrm{pm}(=4 \mathrm{GHz})$ peak-to-peak $(\mathrm{PP})$ on one minute, and $\sim 100 \mathrm{pm}(=41 \mathrm{GHz}) \mathrm{PP}$ on one hour, without active stabilization of the cavity length (Figure 8 ). The laser line is even more stable with the concave output coupler, with 
residual fluctuations of $\sim 6 \mathrm{pm}(=2.5 \mathrm{GHz})$ PP on a $1 \mathrm{mn}$-scale, and $18 \mathrm{pm}(=7 \mathrm{GHz}) \mathrm{PP}$ on one hour (Figure 9). The lower wavelength stability of the plane-plane cavity can be attributed to its strong dependency to the pump power, and to the pump power fluctuations [5].

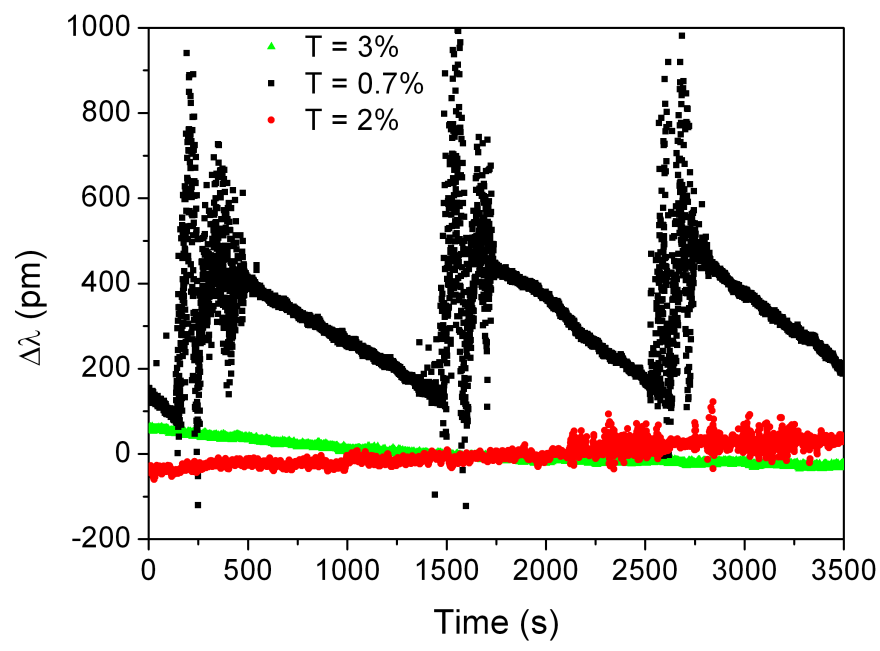

Figure 8: Wavelength variation as function of time for different output couplers in the plane-plane configuration.

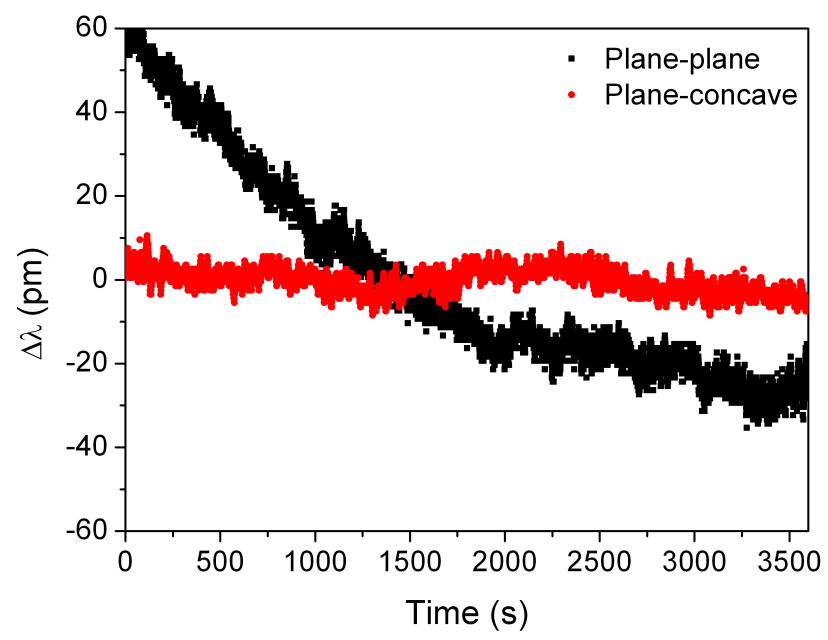

Figure 9: Wavelength variation as function of time for the two configurations studied with a $3 \%$ transmission output coupler.

\section{CONCLUSION}

We have investigated the single-frequency operation of a short-cavity OPS-VECSEL emitting at $852 \mathrm{~nm}$, dedicated to Cs atom spectroscopy. The single-frequency dynamics and the pump-induced thermal lens have been taken into account, to determine the optimum range of operation of the laser source. Experimentally, we have demonstrated a compact semiconductor laser emitting at $852 \mathrm{~nm}$ in single-frequency operation with more than $100 \mathrm{~mW}$ without intracavity elements. Stable single-frequency emission is demonstrated for output coupler transmissions higher than $2 \%$, with no evidence of mode hopping for hours. Using a plane concave configuration the laser stability and the wavelength fluctuation is significantly decreased, below $6 \mathrm{pm}$ on one minute, without active stabilization of the laser cavity. Further work to lock the laser line on a Cs atomic transition is in progress. 


\section{ACKNOWLEDGMENTS}

This work was supported by the French Agence Nationale de la Recherche (ANR-07-BLAN-0320-03). F. Camargo was funded by the RTRA/Triangle de la Physique (2010-089T).

\section{REFERENCES}

1. Klehr, A., Wenzel, H., Brox, O., Bugge, F., Erbert, G., Nguyen, T-P. and Tränkle, G., "High-power 894 nm monolithic distributed feedback laser", Opt. Exp 15(18), 11364-11369 (2007).

2. Gilowski, M., Schubert, Ch., Zaiser, M., Herr, W., Wübbena, T., Wendrich, T., Müller, T., Rasel, E.M., Ertmer, W., "Narrow bandwidth interference filter-stabilized diode laser systems for the manipulation of neutral atoms" Opt. Commun. 280, 443-447 (2007).

3. Kuznetsov, M., Hakimi, F., Sprague, R., Mooradian, A., " Design and characteristics of high-power $(>0.5 \mathrm{~W}$ CW) diode-pumped vertical-external-cavity surface-emitting semiconductor lasers with circular $\mathrm{TEM}_{00}$ Beams", IEEE J. Selec. Topics in Quantum Electron. 5, 561 (1999).

4. Jacquemet, M., Domenech, M, Lucas-Leclin, G., Georges, P., Dion, J, Strassner, M., Sagnes, I. and Garnache, A., "Single-frequency $\mathrm{cw}$ vertical external cavity surface emitting semiconductor laser at $1003 \mathrm{~nm}$ and $501 \mathrm{~nm}$ by intracavity frequency doubling", Appl. Phys. B 86, 503-510 (2007).

5. Laurain, A., Myara, M., Beaudoin, G., Sagnes, I. and Garnache, A., "Multiwatt-power highly-coherent compact single-frequency tunable Vertical-External-Cavity-Surface-Emitting-Semiconductor-Laser”, Opt. Exp. 18(14), 14627$14636(2010)$.

6. Hastie, J., Hopkins, J-M., Calvez, S., Jeon, C.W., Burns, D., Abram, R., Riis, E., Ferguson, A.I., Dawson, M.D.; "0.5-W single transverse-mode operation of an 850 -nm diode-pumped surface-emitting semiconductor laser",. IEEE Photon. Technol. Lett. 15(7), 894-896 (2003)

7. Cocquelin, B., Holleville, D., Lucas-Leclin, G., Sagnes, I., Garnache, A., Myara, M. and Georges, P., "Tunable single-frequency operation of a diode-pumped vertical external-cavity laser at the cesium D2 line", Appl Phys B 95, 315-321 (2009).

8. Laurain, A., Myara, M., Beaudoin, G., Sagnes, I., Garnache, A., "High power single-frequency continuously-tunable compact extended-cavity semiconductor laser", Opt. Exp. 17 (12), 9503 (2009)

9. Garnache, A., Ouvrard, A., Romanini, D., " Single-Frequency operation of External-Cavity VCSELs: Non-linear multimode temporal dynamics and quantum limit", Opt. Express 15, 9403 (2007)

10. Chénais, S., Balembois, F., Druon, F., Lucas-Leclin, G. and Georges, P., "Thermal Lensing in Diode-Pumped Ytterbium Lasers-Part I: Theoretical Analysis and Wavefront Measurements", IEEE J. Quantum Electron. 40, 12171234 (2004).

11. Kemp, A., MacLean, A., Hastie, J., Smith, S., Hopkins, J.-M., Calvez, S., Valentine, G., Dawson, M., Burns, D., "Thermal lensing, thermal management and transverse mode control in microchip VECSELs", App. Phys. B, 83, 189 (2006)

12. Siegman, A. E. [Lasers], University Science Books, California, 747 (1986). 